\title{
POINT SYMMETRY IN PLANETARY NEBULAE
}

\author{
ROMANO L.M. CORRADI and HUGO E. SCHWARZ \\ ESO, Casilla 19001, Santiago 19, Chile \\ and \\ LETIZIA STANGHELLINI \\ Osservatorio Astronomico di Bologna, via Zamboni 15, Bologna, Italia
}

We present narrow band images and spectra illustrating the morphological and kinematical characteristics of a new class of planetary nebulae. It consists of all those objects in the catalogue by Schwarz et al. (1992) whose morphology shows no other symmetries than point-reflection about the central source. This peculiar shape is often determined by pairs of ansae or blobs separated from the central body of the nebula. The kinematical data show both low (e.g. IC 4634) and high (He 2-186; $V_{\text {exp }}>135 \mathrm{~km} \mathrm{~s}^{-1}$ ) velocity outflows.

The peculiar property of the pairs of blobs is that: a) the radially opposed blobs in a given pair are red, respectively blue shifted, and b) the different pairs can have reversed shifts. This means that red and blue shifted material is seen on the same side of the central nebula.

We explain these properties as being the result of multiple mass ejections from interacting binary systems composed of a hot star, accreting matter from a masslosing primary, and forming an accretion disk. The observed blobs then are the result of discrete mass ejection events. The relative blue/redshifted patterns are caused by precession of the disk driving the mass outflow from the system. The observed morphology and kinematics are then explained by projection of the precession cone onto the plane of the sky.

\section{References}

Schwarz, H.E., Corradi, R.L.M., and Melnick, J. 1992 AASS, in the press 